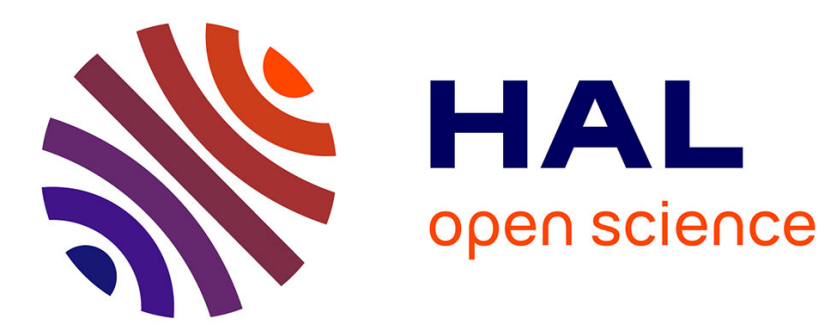

\title{
Notes sur C. CINO SENOVIRI
}

Colette Bémont

\section{To cite this version:}

Colette Bémont. Notes sur C. CINO SENOVIRI. Gallia - Fouilles et monuments archéologiques en France métropolitaine, 1971, 29 (2), pp.200-217. 10.3406/galia.1971.2577 . hal-01934479

\section{HAL Id: hal-01934479 https://hal.science/hal-01934479}

Submitted on 11 Mar 2020

HAL is a multi-disciplinary open access archive for the deposit and dissemination of scientific research documents, whether they are published or not. The documents may come from teaching and research institutions in France or abroad, or from public or private research centers.
L'archive ouverte pluridisciplinaire HAL, est destinée au dépôt et à la diffusion de documents scientifiques de niveau recherche, publiés ou non, émanant des établissements d'enseignement et de recherche français ou étrangers, des laboratoires publics ou privés.

\section{(이) $\$$}

Distributed under a Creative Commons Attribution - NonCommercial - NoDerivatives| 4.0 


\title{
Notes sur C. CINO SENOVIRI
}

\author{
par Colette BÉMONT
}

Ine enquète récente m'avait amenée i identifier un potier, dont les signatures manuscrites -... partiellement et mal conservées, le plus souvent -- soulignaient le décor de bols I). 37 : C. C:INO SEVOVIR... I.'étude d'une série de cinq fragments a permis de déchiffrer l'essentiel du nom, a révélé un répertoire comprenant déja quatorze motifs et mis en évidence, chez l'artisan, la fidélité -.. probable ou certaine pour le matériel considéré ... a un mode de composition du décor à métopes ${ }^{1}$. Plusieurs questions, cependant, se posaient encore. En effet. la forme de la signature : C. CINO SE.VOIIR..., composée apparemment d'un praenomen et de deux cognomina d'origine celtique, trahissait le "barbare" romanisé, mais, en mème temps. paraissait peu compatible avec les usages gaulois : le double cognomen n'avait aucune justification ${ }^{2}$. Toutefois le pietre état de l'unique tesson qui nous avait fourni la forme la plus complete du second cognomen ${ }^{3}$ nexcluait pas la possibilité que l'inscription

1 Signature inedite sur un moule de sigillex : d. CINO SEXOV..., dans fallia, XXV1, 1968, p. 301313; Ln potier ou decorateur du Sud de la riaule, dans (iallia, X.5VII, 1969, p. 186-205).

2 Signature inédite..., p. 306-30x.

\& I, bol I) conserve a Saint-Remy-de-Provence In potier..., p. 189 +1 190. tig. 3). continuàt au-dela de la derniere lettre lisible. le $K$, et que de nouveaux fragments pussent éclairer le texle de façon satisfaisante et indubitable a la fois. Il était probable, en outre, que nous ne possédions pas. avec ces maigres débris, l'ensemble des poinçons décoratils de CINO el certain, en tout cas, que les oves faisaient défaut. Cette lacune était d'autant plus regreltable qu'ils avaient des chances d'apporter la preuve que le décorateur. utilisant, par ailleurs. des types figurés communs. pour la plupart. a la (iraufesenque et à Banassac. avait travaillé dans l'un plutôt que dans l'autre de ces centres. Entin, il importail de voir si la série que nous possédions révélail une technique immuable ou si elle presentail seulement l'un des modes de composition utilisés par Cilvo.

Quatre nouveaux fragments se sont ajoutés depuis lors au matériel connu : deux d'entre eux proviennent des bords de la Méditerranée. aux confins de la France el de l'Italie. le troisieme de l'embouchure du Rhònes, le

4 1.e lesson is ne porte que liextremite diun pendentif peu caracterise of un fragment darceau.

¿ les deux premiers mont ile signales par (i. B. Hogers. I.un $F$ dans ce cataloguej, qui provient de legolise de Beaulieu-sur-Der Alpes-Daritimes, se Iroure au muser de Wenton; lautre \&, découvert an cours des foulles d'Albintimilium of depose au 


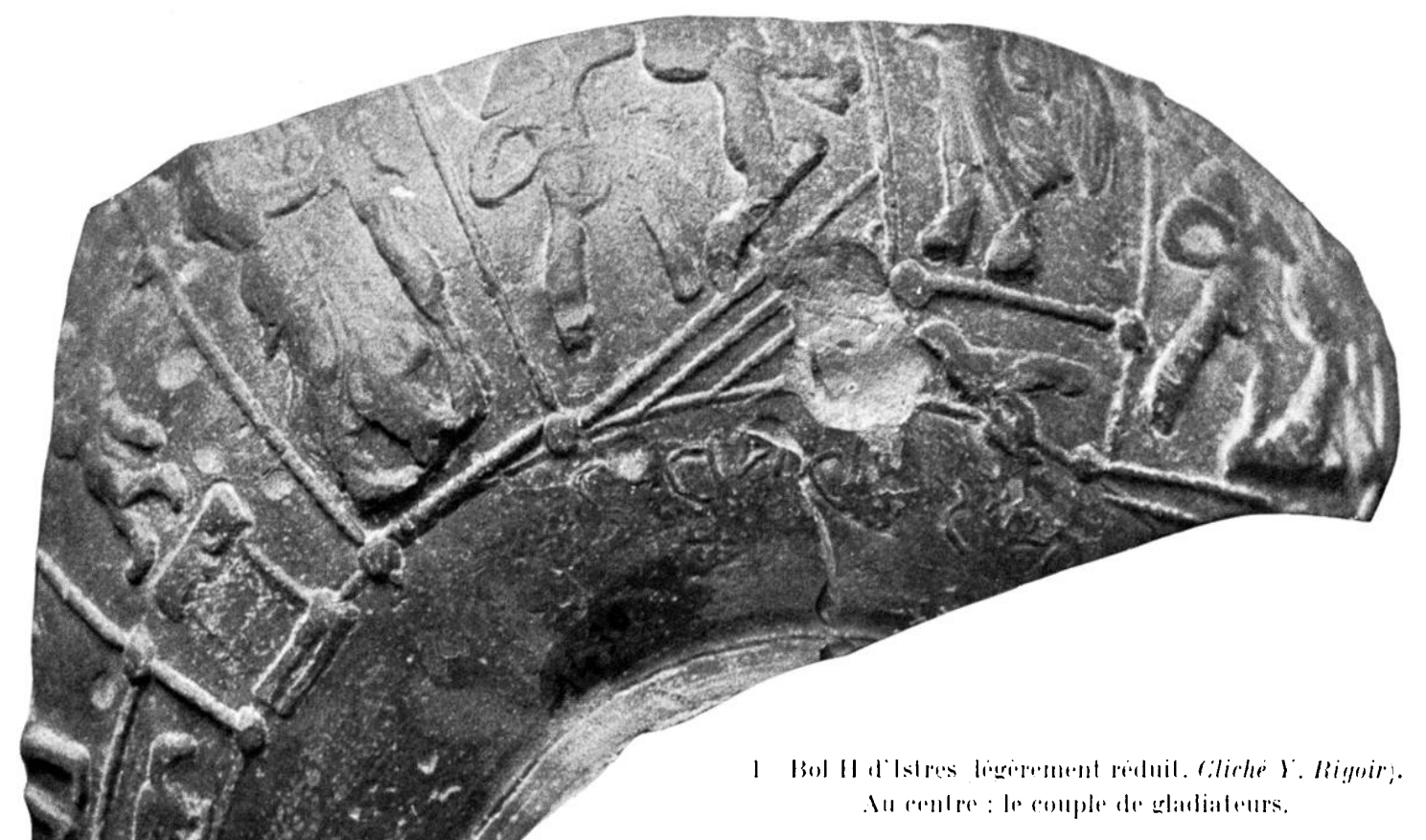

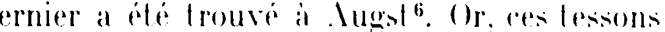
contribuent soit a confirmer les résultats dejai obtenus. soit à laire progresser l'enguele.

musere de Vintimille, a de publie par X. Iambontia (Gli scavi di . lbintimilium e la cronologia della ceramica romana. Campagne di searo 1938-19.10, Bordighara,

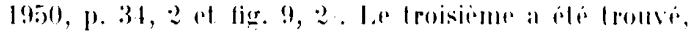
voilà quelques anmeres, à Fos-sur-llar Bouches-du-

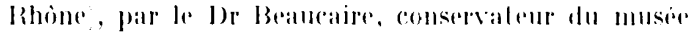
d'Istres. Ia presence de ce tesson H ma ble signaler.

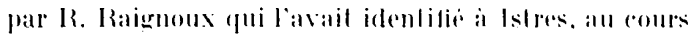
le liate 1969 .

Je remercie, ici, 19 m. Salm, conservatrien du musier de Menton et le Dr Braucaire, qui mont laissi si courtoisement toule diberte de disposere des tessons qui ont servi a collo ifudr.

6 Il a bli publie par E. Elllinger (l)ie heramil:

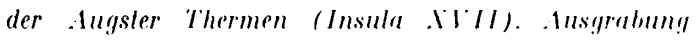
19.3\%-38, Monographien zur I r- und lrühgeschichle der schere, R. VI, Batle, 1949, pl. 15, 176, 1, 6xi. Je dois a lamabilite de lantent la photographere du tesson ici reproduite.

lous. romme les précédents. ne comportent quine partie de l'inseription originelle. L'attribution de trois d'entre eux est. d'emblee hors de doute : le bol II, d'Istres, qui conserve un long morceau de la signature. C. CIINO SE.V ... fig. 1 et 2. 2, 3i. le bol F. de Venton. marque $(\therefore$ C CIN o... (lic. :3, 3-j) el presenlant, dans son décor. des poinçons communs a plusieurs vases de CiLO SE.VOITR . . fig. :3. $l^{7}$ : le fragment I. d'Augst qui porte une aulre partie de lonscription : ..o SE.VOII .. sous les restesde lons metopesoreupées par des poincons ailleurs utilises. l'autel et la touffe Wherbe fig. 1. 3.8. Ce dernier. qui semble ne rien nous apprendre sur le polier. a. au moins. le mérite de ronfirmer indisculablement l'altrihution du lesson C de saint-Remy-de-Provenee fig. 4. I . en prouvant que les vases de C. C:I.TO

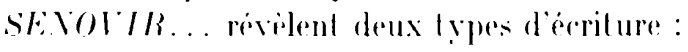
l'une réguliere el menue. l'aulre aussi soignée mais plus exuberante et caracterisé par des

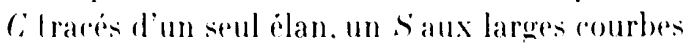
dépassant l'alienement au-desisus et au-dessous:

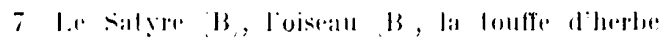

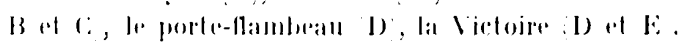
ef. In potier..., tig. $8,1,2, t, 11,9$, sams rompter les trois antres fragments.

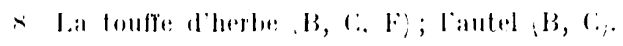




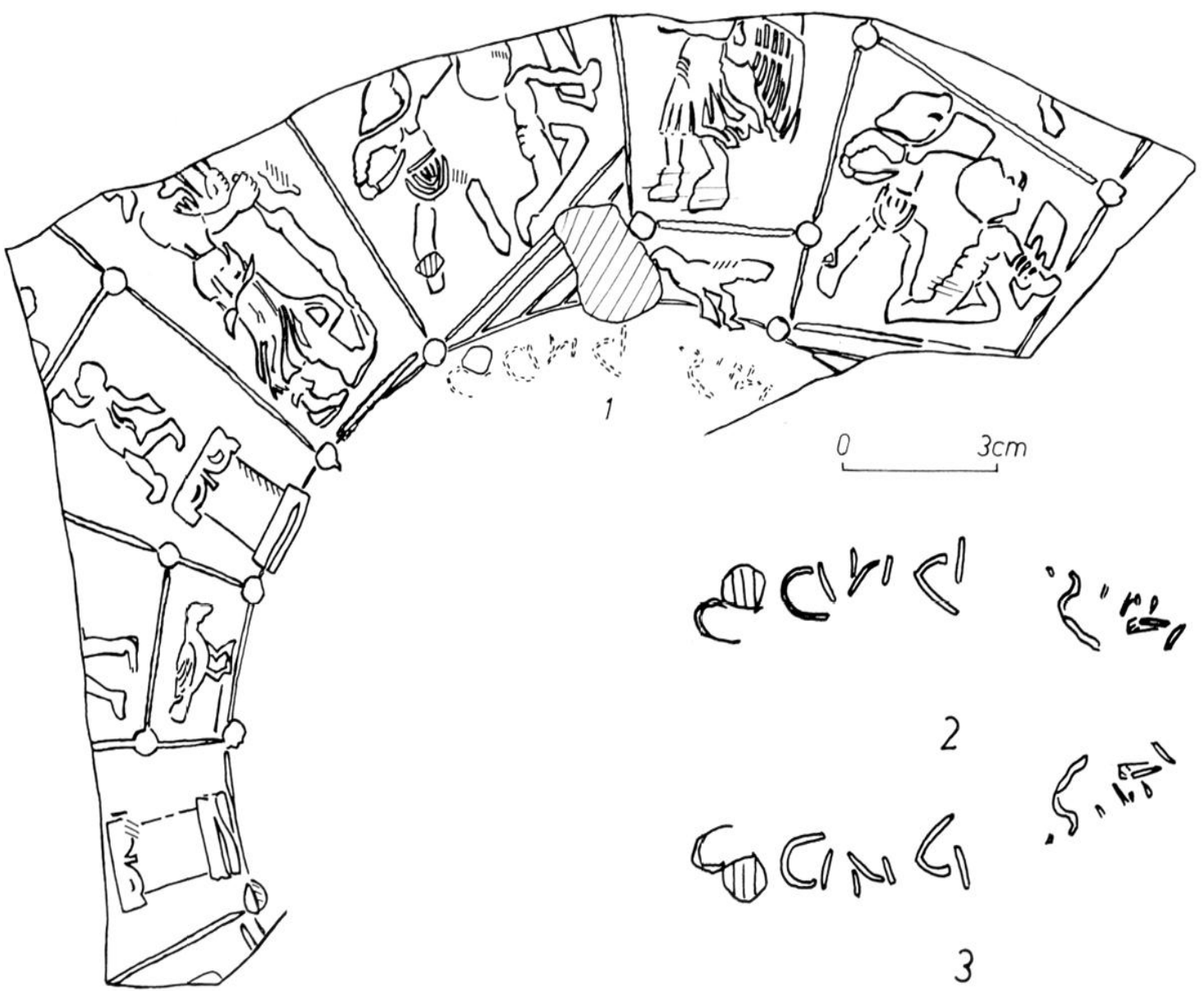

2 Bol 11 d'Istres. 1, décor; 2, signature : fac-simile telle quion la roit, ; 3, signature: fac-simile (telle quion la lit ech. I: 1 .

et des $X$ au ductus plus hardi, dépassant, eux aussi, le corps des autres lettres (fig. 4, 2,4). Le dernier tesson, le bol ( $\mathrm{i}$ de Vintimille, a été daté de la fin du ${ }^{\text {er }}$ siècle et attribué au Surl de la Gaule par X. Lamboglia. qui est. toutefois. demeuré perplexe devant la signalure. réduite rette fois a la plus grande partie de son dernier élément. Celte suite de lettres n'a semblé. d'abord, ì X. Lamboglia qu'avoir un rapport tres lointain, pour ne pas dire nul. aver le nom que je croyais distinguer d'apres la publication?. Mais le frottis que l'Institut d'études ligures a bien voulu me fournir ${ }^{10} \mathrm{~m}$ 'a

9 Gli scavi..., ioc cil. et lell'r' du 11 decembro 196!) du Professeur Lamboglia.

10 Il ne ma pas ete possible. Iors d'un vovage a Vintimille, davoir acces a ce vase et je dois a lobli- incitee plus encore a reconnaitre ... $I I N O$ $V I R I=\ldots E X O I I R I$ et R. Marichal, a qui j'ai soumis le document, a confirmé ma lecture : nous avons ici la fin d'un mot, qui sachèce sur un $I$ long et qui ne peut ètre que ... ENOIIRI (fig. 5), 2-3). Or, on ne connait pas. par les publications actuelles, d'autre potier signant identiquement. sous un décor ì métopes. et dont le nom présente la même forme. De plus, les poinçons figurés du bol de Vintimille appartiennent au répertoire connu de $C I N()$ : c'est-a-dire que nous avons affaire non seulement aux mèmes types, mais à des variantes identiques de reux-ci. En outre, le morceau conservé du bol révicle une organisa-

geince de (i. B. Rogers les relevés qui mont permis de completer le frotlis de l'Institut detudes ligures. 

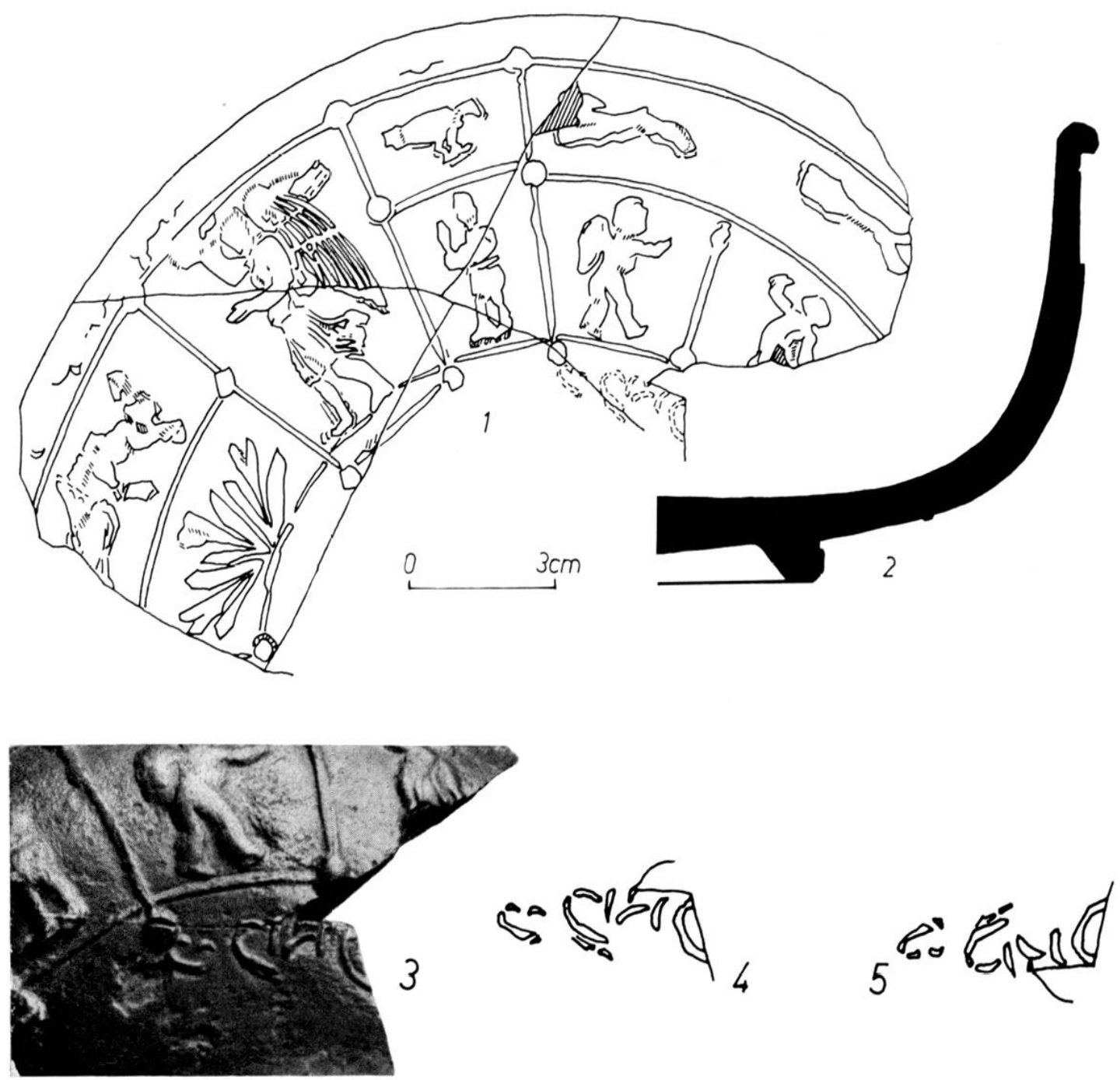

3 Bol F de Menton. 1, decor ; 2 , profil ; 3, signature : photographie; 4 , signature : fac-simile telle qu'on la voit!; i, signalure : fac-simili telle quion lat lit, ech. $1: 1$ ).

tion symétrique des métopes de part et d'autre du panneau de la Victoire et l'occupation de ces cadres, qui se répondent. par des motifs identiques et orientés dans le mème sens. Enfin mème si l'exécution n'est pas parfaite ... on constate, en effet. une certaine irrégularité dans les proportions des deux métopes de mème contenu - - la différence de taille entre ces dernières et le panneau de la Victoire et la similitude de largeur, au contraire. entre cette métope et celle de la IDiane permettent d'imaginer un systeme de correspondance comparable à celui que nous avons analysé dans une précédente étude ${ }^{11}$ (fig. $6,2,1$ ). Il parait donc assez difficile d'attribuer le fragment de Vintimille -... compte tenu, par surcroit, de la datation qu'on lui assigne depuis longtemps à un autre qu'à C. CIINO. L'un des intérèts de l'identification est de fournir la fin de la signature : nous lisons là, sans les hésitations qu'inspirait l'inscription du bol $\mathrm{J}^{12}$, un $I$

11 Si l'on retablit, hỵpothétiquement, le décor, d'apres le schema des bols $B$ et E, on voit que les trois métopes semblables entre elles qui feraient suite au panneau de la Diane auraient les mèmes proportions que, sur les premiers bols, leurs analogues fig. 6,2 .

1.2 In potier..., loc. cil. 

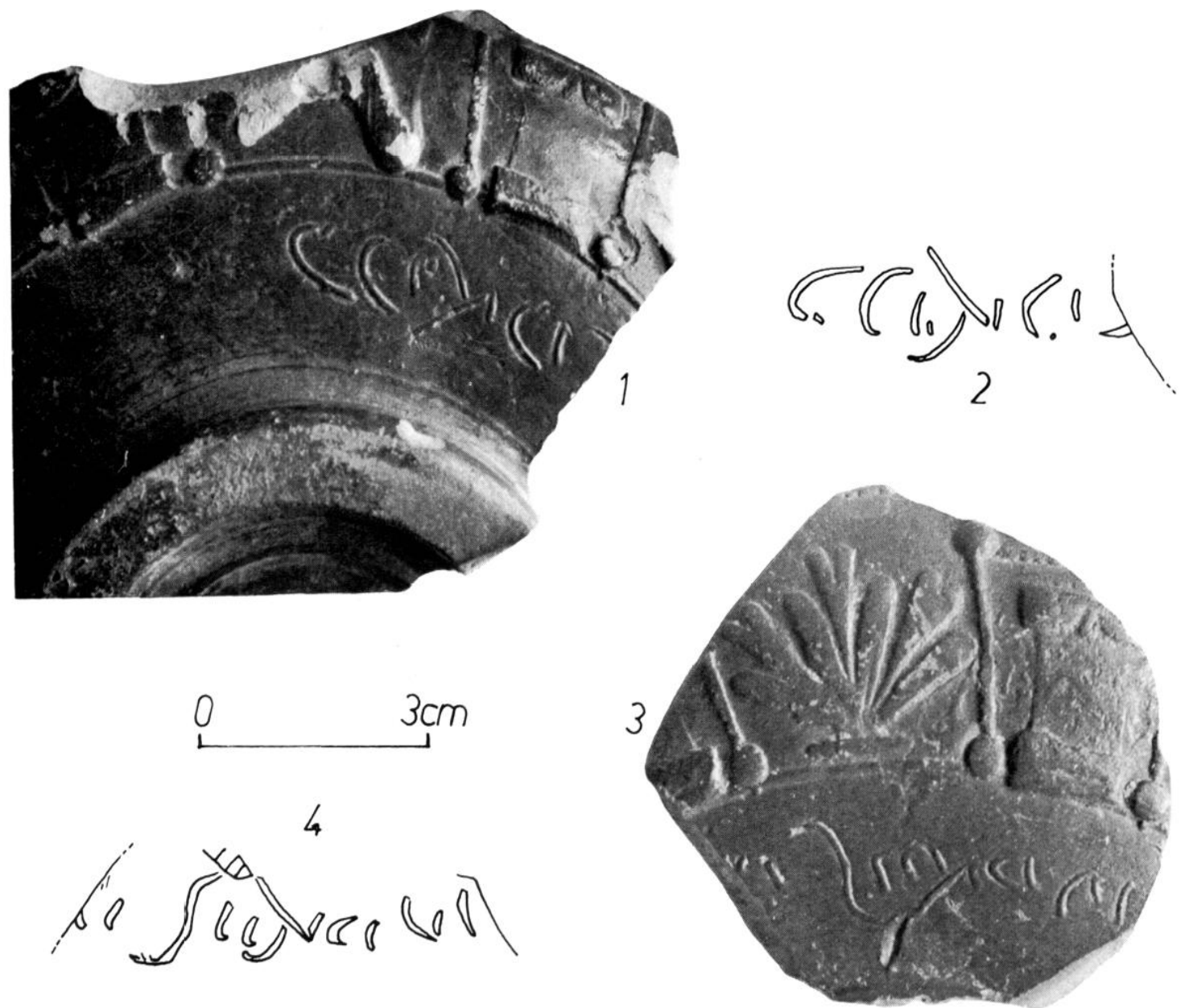

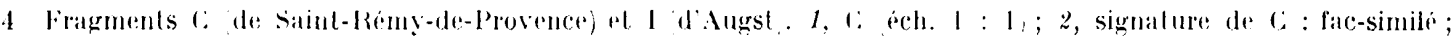
3, I iech. I : l); t, signature d'I : fac-simile. Notar que les parties commumes des signatures paraissent se superposer.

apris le R. Linsi le probleme posé par le seeond cognomen paraìt résolu : il s'agril d'un génilif. Et romme $(: I . V)$ ne semble pouvoir ètre qu'un nominatif's SEVOITR(OS) est, selon loute apparence. soit le patron. soit le pere de $C$. CINO. Il est diflicile de trancher a priori entre les deux hypotheses. élant domme le raratelere insolite de celte forme de signature. mais la seconde est, a mon avis. la plus justifiée. la dépendance, en eflel. n'est d'ordinaire indiquée que pour un esclave a l'exard de son maitre. Or, C: CINO) parait bien l'embryon d'un nom romanise sur te modiale des lria nomina du ritoven. Ionr. a supposer quion

1.3

Signature inedite..., 1). 305-3066. admetle re qui n'est pas loujours le ass l'existence d'un systeme d'esclavage dans les entreprises gauloises, on conviendra que le nom C. C:INO, loin d'offrir aucune indication qui permelle de reconnailre au moins selon les normes habiluelles un individu réduit a

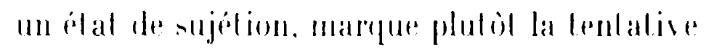
d'un homme libre pour adopler les roulumes romaines. Faire de SESTOI IR(OS) le prere de (C. (IN) ne présente pas de difficultés insurmontables : si ce lype de signature parait alre une nouveaule par rapport a ce quion roit sur les moules ronnus. il nest pas rare, en revanche. dans les inscriptions. quiun gaulois. surtout assimile a la premiere generation. indique sa filialion par le génitif du nom unique selon 

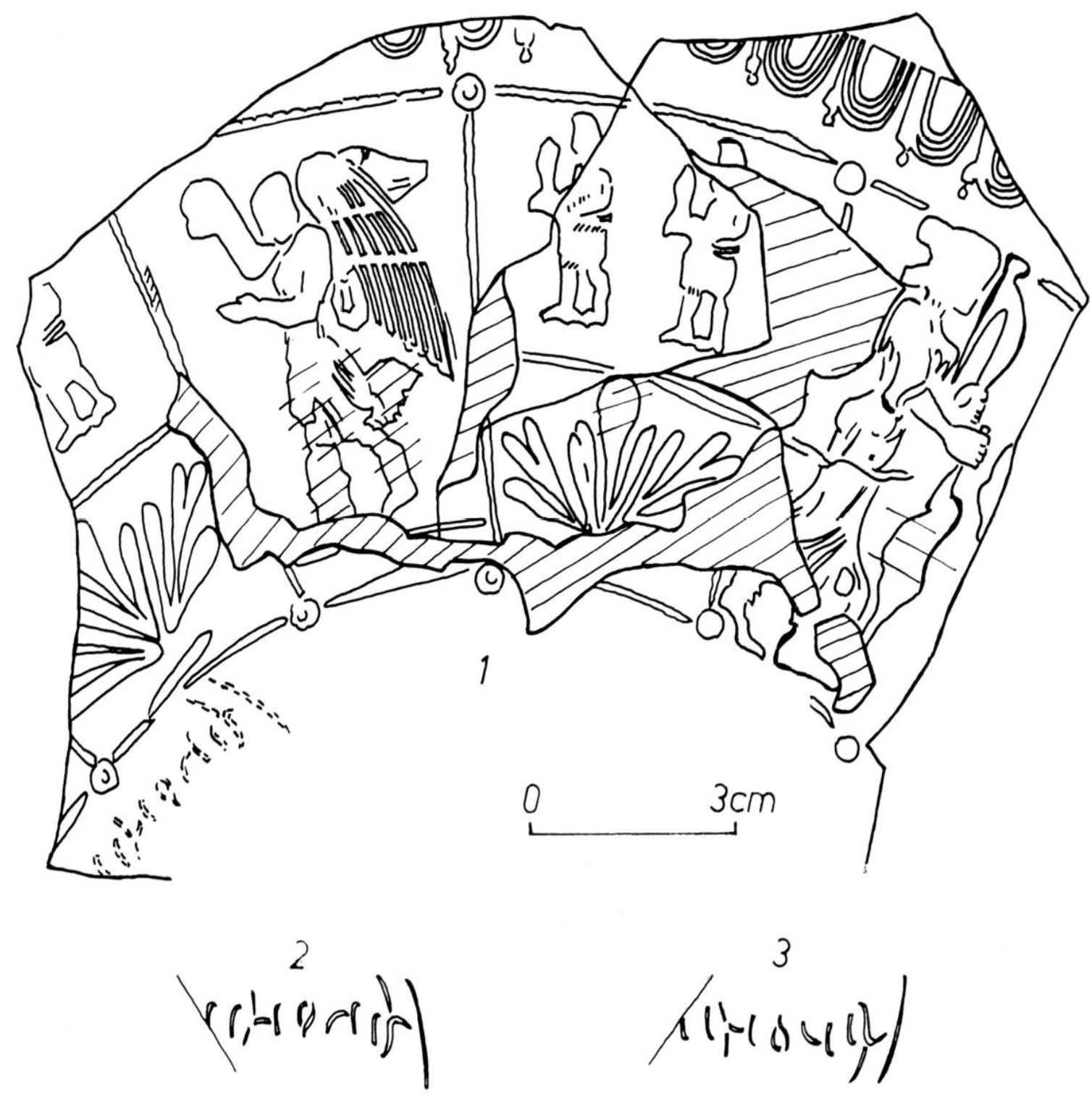

j Bol G de Vintimille. 1, décor; 2 , signature : fac-simile telle quion la voit;; 3, signature : fac-simile ; lelle qu'on la lit i.

l'usage local ou du cognomen de son pire'14. I)onc, à moins de supposer - c- ce qui est tris improbable -.- que $C I N O$ est la forme abrégée

14 On peut être certain, parfois, que le père portait un nom unique : par exemple lorsque le gentilice du fils est directement dérivé du nom à un seul élément du pere cf. CII. XIII, Index : notabilia varia. En revanche, une dissemblance totale entre le nom unique du pere et les nomen et cognomen de son fils interdit de savoir si le premier, designé simplement par un cognomen, son signe distinctif, ne portait pas la' même gentilice que te second. (sans nécessité) d'un gentilice lui-mème au génitif ${ }^{15}$, il y a tout lieu de penser que nous lisons sur les neuf bols la signature de $C$. CINO fils de SEVOVIR(OS). SENOVIR(OS) étaitil le potier dont on signale des marques sur vases lisses a Orange et à La Graufesenque ${ }^{1+}$ ? C'est ce que je ne saurais dire.

15) Signature inédite..., ibid. La disparité des cas, qui semble ici evidente, exclut l'utilisation, comme gentilice, du cognomen en -o/-onis, altestee ailleurs tres exceptionnellement.

16 C:IL. XII, 5686, 816; cf. Un potier..., p. 190, 
Le décor, du double point de vue de la structure et des molifs utilisés, ne présente pas. pour chacun des nouveaux bols, le même intérêt. Le tesson I d'Augst pose un petit problème, car je ne peux exclure absolument l'éventualité qu'il soit issu du mème moule que le fragment $\mathrm{C}$ de saint-Rémy-de-Provence. 'Toutefois les comparaisons que j'ai lailes entre les deux échantillons ne sont pas concluantes, car elles ne portaient que sur la surface décorée. non sur le profil, et la partie qui pourrait ètre commune aux deux décors est extrêmement réduite. Jans cetle incertitude on doit accepter une alternative. si C et I sortent du mème moule, la construction du vase, équilibrée par rapport à des axes de symétrie, diflére en deux points, au moins, du schéma illustré déjà par les bols B. I), E : il n'y a pas de correspondance entre la taille de la métope au guerrier. ou passe l'un des axes, el celle de la métope à la touffe d'herbe (fig. $\mathbf{6 , 4}$ ); le même motif (l'autel) est répété en des endroits différents du mème quart de décor ${ }^{17}$. Si, en revanche, $\mathrm{C}$ et I sortent de deux matrices différentes. l'ornementation du vase I est compatible, elle aussi, avec un schéma décoratif équilibré par rapport a deux axes perpendiculaires et l'un d'entre eux peut traverser la métope à la touffe d'herbe. Cette hypothèse parail pour le moment la plus vraisemblable, mais seule la comparaison directe des deux pieces permettra de trancher la difficulté (fig. 6.j). Le vase $\mathrm{G}$ de Vintimille, nous l'avons vu, est sûrement orné suivant les mêmes principes de symétrie que B, I), E et peut également être décoré selon le schéma connu. fondé sur la succession rythmée de huit métopes pour chaque moitié de la panse. Les irrégularités dans la mise en ouvre n'autorisent pas a concevoir sur ce point une absolue certitude, mais cette hypothese, compte tenu des intentions apparentes de l'artisan. est très vraisemblable et, en tout ras, la faible

192 et 193. La presence de signatures contenant la forme SENOVIRI sur des vases lisses trouvés à la Graufesenque ma ete signalec par I.. Balsan.

17 Sur les vases assez largement conserves, te même poinçon, à en juger du moins d'après les metopes qui subsistent, n'apparait qu'une fois dans la séfuence comprise entre deux axes de symetrie ef. I'n potier.... fig. 6,3 et 7 ; surface du fragment interdit d'imaginer, avec un minimum de précision. un mode différent de construction. Le bol II d'Istres, pour sa part, ne pose aucun probleme : largement conservé, au moins dans sa partie inférieure, le décor se conforme de façon quasi parfaite aux rogles de construction observées sur B, D, E et il relive. dans celte famille, de la mème variété que le bol 13 : les panneaux découpés sur la surface de la piece sont de deux largeurs différentes (fig. (i, 3), alors que $\mathrm{D}$ ) et $\mathrm{E}$ présentent trois types de mélope distincts ${ }^{18}$.

Le bol F. de Menton, tout en se rattachant indubitablement à une série. présente quelques traits originaux. Le hasard, d'abord, a fait que le profil entier fùt conservé (fig. 3, 2). On constate done l'existence d'une lévre saillant nettement vers l'extérieur, arrondie au-dessus, aplatie en dessous. Ie pied se distingue, si on le compare a la pluparl des autres exemples, par une assise moins large, un bourrelet extérieur peu marqué, une gorge fine. à peine indiquée ${ }^{19}$. I a palte dure. bien cuite, l'engobe brillant classent ce vase, du point de vue strictement technique, parmi les plus réussis de la série (aver C et I)). Mais c'est le décor qui comporte les particularités les plus intéressantes. Car l'examen du tesson révèle une composilion necessairement différente de celle des autres exemplaires : il reste à savoir jusqu'où est poussée la diversité. Fn effet, alors que nous possédons plus du tiers de la zone décorée. le morceau se décompose en quatre métopes presque complites el le contenu de chacune est différent, comme sont différentes leurs largeurs respectives. Ainsi, nous ne trourons plus les systimes de correspondance -... fondes sur le partage d'une portion analogue de la panse en cinq panneaux -. qu'avaient fait apparaitre trois des bols précédemment analyses (B. E et II) el que semblaient reproduire un, voire deux autres vases (I) et ( $i$ ?) (fig. (6. 1$)^{20}$. l)e plus, indépendamment des détails dans l'économie de la surface, rien, au

If Ibid.

19 ces particularites lo differencient de formes precedemment itudiees lin potier..., p. 193, fig. 5, 3, $t, j$, que caractirisaient un bourrelet exterieur saillant of une large assise plate.

20 Ibid., fig. 6, 3 e. 7,4 ; ci-contre, fig. 6, 2 . 


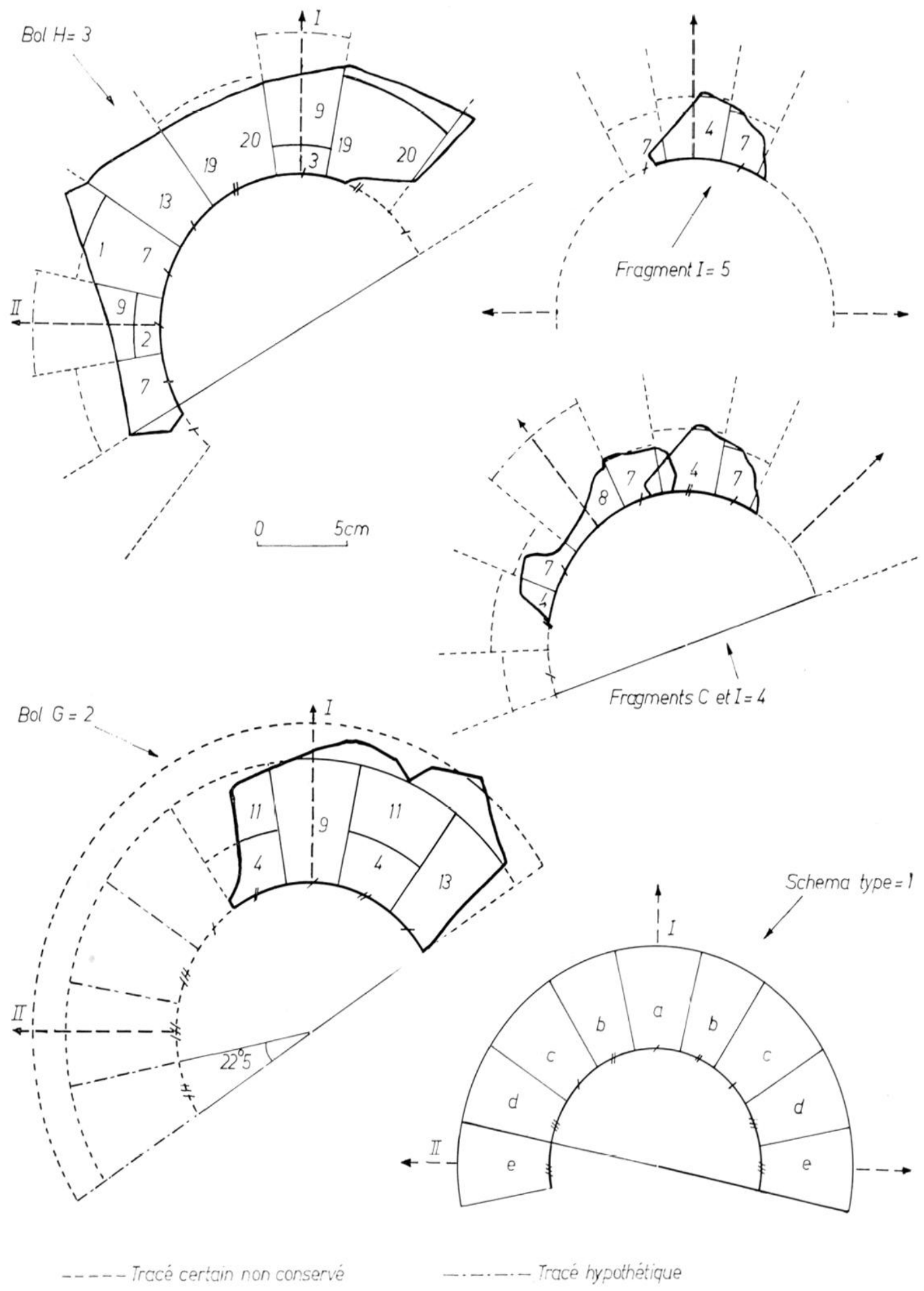

(6) Composilion dies decors (i, H, I. 1, schima de lorganisation des metopes dapres les bols B et $\mathrm{E}$, reproduetion partielle de la zone dicorie ; 2 , restitution hypothetique de la disposition du decor de $6 ; 3$, le bol $\mathrm{H} ; 4$ et 5 , hypotheses relatives all fragment I 4 : l et I sortent du mème moule et se completent; ; : I, appartenant à un modile different de c, admet un axe de symetrie). 
premier regard, ne permel mòme de concevoir une construction d'ensemble supposant une quelconque harmonie. L'hypothese d'une complete fantaisie, loutefois, résiste mal a la ritique : le colé mécanique et assez simpliste de tels décors exige - ne serait-ce que pour la commodité de l'artisan - une division équilibrée de la surface et, de fait, les fragments de sigillée, de meme style. que j'ai pu examiner ont révélé - pour peu qu'ils fussent assez étendus. ou heureusement silués ... l'existence, pour chaque vase, au moins d'un partage symétrique. C. CINO nous le savons. use, en d'autres cas. d'un systime simple et assez rigide pour diviser la surface el meubler les métopes. Il serait donc fort étonnant qu'il fît preuve, dans une situation analogue, d'une grande originalité. sans nécessité pratique aucune. Aussi y a-t-il des raisons de postuler, a litre d'hypothèse. l'usage d'une répartition symétrique des panneaux sur les flanes du bol F. Trois éventualités soffrent alors : une symetrie par rapport a un axe extérieur à notre fragment, ou intérieur a celui-ci, ou encore une symetrie par rotation des deux moitiés du vase.

Imagriner un axe exlérieur à notre lesson implique la répétition, de part el d'autre de l'axe, a égale distance, des mèmes métopes, pareillement occupées. Ce qui entraine un déséquilibre inesthétique de l'ornementation. a moins d'une identilé de tous les panneaux du vase, ou, au moins, d'un partage subsidiaire harmonieux (et lui-mème symétrique) de charune des deux moitiés du décor. Or, dans le cas qui nous occupe, la longueur de la séquence conservée et la disparite de toutes les métopes qui la composent interdisent, quelle que soit la place attribuée a l'axe extérieur, de rétablir une heureuse organisation de chaque moitie du vase (fig. 7,1 ) : rette solution parait donc a ecarter.

En revanche, on peut aisément concevoir la répétition, dans le mème ordre. de deux séries de panneaux diamétralement opposés par rapport au centre de la zone circulaire décorée (fig. 7. 2). C'est le minimum qu'autorise à imaginer, logiquement, la suile de quatre métopes que nous possédons. Mais, on ne saurait, admettre toulefois l'usage de trois groupes identiques a ce fragment : il représente, nous l'avons vu, plus du liers de la surface à décorer.

supposer, enfin, la présence d'un axe de symétrie intérieur implique, puisque celui-ci n'est pas immédiatement apparent, qu'il passe au milieu d'un des panneaux occupant les extrémités du tesson. Il est nécessaire, également. à l'équilibre de l'ornementation que chacune des moiliés du décor comporle une symétrie interne. Faisons l'épreuve de cette construction : l'atlention est d'abord altirée par la grande métope contenant le petit satyre et l'Amour. I a surface du panneau est incompliele, mais l'opposition, face à face, des deux figures, de part et d'autre de la colonnette, exclut l'existence d'un troisième personnage au-delà du salyre. De plus, le groupe qui occupe le registre supérieur un lière poursuivi par un chien - constilue une scene si traditionnelle qu'on ne saurait rien imaginer derriere le rhien : il est donc justifié de penser que nous avons le contenu complet de celle large métope et que le milieu de celle-ci doit etre, normalement, marqué, entre les figures affrontées, par la colonnette. Essayons de tracer l'axe de symétrie supposé, c'est-à-dire le diamitre du cercle de base du décor qui passe par le pied de la colonne: nous constatons qu'un autre diamelte du mème cercle, mené perpendiculairement au premier, partage juste en son milieu la limite inférieure du panneau qui contient la louffe d'herbe. Nous avons mis ainsi en évidence l'existence possible de deux axes de symétrie complémentaires qui traverseraient. la premiere el la dernière métopes de ce fragment et partageraient, chacun, en deux parties égales chacun des demi-cercles ...- c'esta-dire chacune des moitiés de décor -- déterminés par l'autre. Il n'y a donc, d'un point de vue crémétrique, aucune difficulté à reconstituer l'ornementation de ce vase en quatre parties semblables, se répondant deux a deux de part et d'autre de deux axes de symétrie orthogonaux, passant, chacun, par le milieu de deux métopes identiques et opposées (fig. 7, 3). L'équilibre du décor serail alors exactement. réalisé et l'apparente incohérence du fragment que nous analysons s'expliquerait par le fait que nous avons entre les mains a peine plus que l'un des quatre secteurs délimites par l'intersection des deux lignes idéales de cons- 


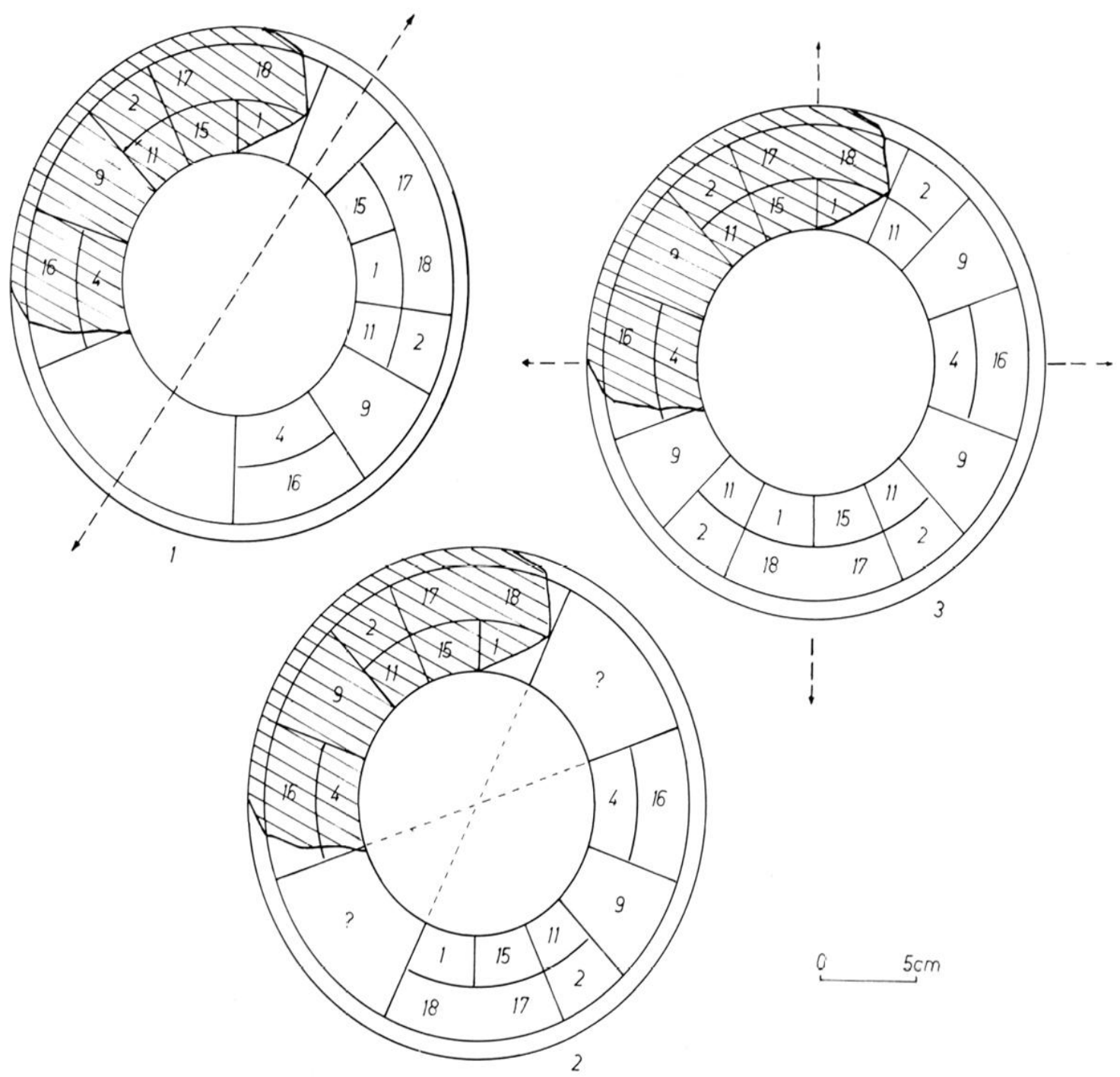

7 Restilutions du decor de F. 1, symelrie impossible par rapport a un axe exterieur au fragment conservé ; 2, répétition de la mème séruence de part et dautre du vase; 3, construction équilibrie par rapport à deux axes interieurs.

truction. Outre une possibilité théorique, il existe, d'ailleurs, quelques présomptions en faveur de celle hypothese : d'autres bols de C. CINO, nous l'avons constalé, sont ornés selon le mème principe général de composition; de plus, l'artisan a l'habitude - signalée déjà el peut-être liée à la succession des opérations auxquelles il se livre de signer à proximité immédiate d'un des axes de symétrie, et l'inscription se trouve, ici. sous la grande métope. Cette recomposition. tris tentante.

:L Ln potier..., p. 196. paraît donc, en mème temps, des plus vraisemblables. Mais si d'autres découvertes venaient infirmer sa valeur, ce serait, tres probablement, pour nous ramener à la deuxieme des éventualités envisagées.

Si nous considérons, maintenant, la nature des motifs choisis, nous constatons qu'elle confirme, en une certaine mesure, les remarques suggrérées par les autres fragments décorés : en effet, les sujets déjà connus se présentent, sur les tessons F, G, H, I, exactement sous la mème forme et orientés dans le mème sens. Mais la fraction conservée des vases ne permet 

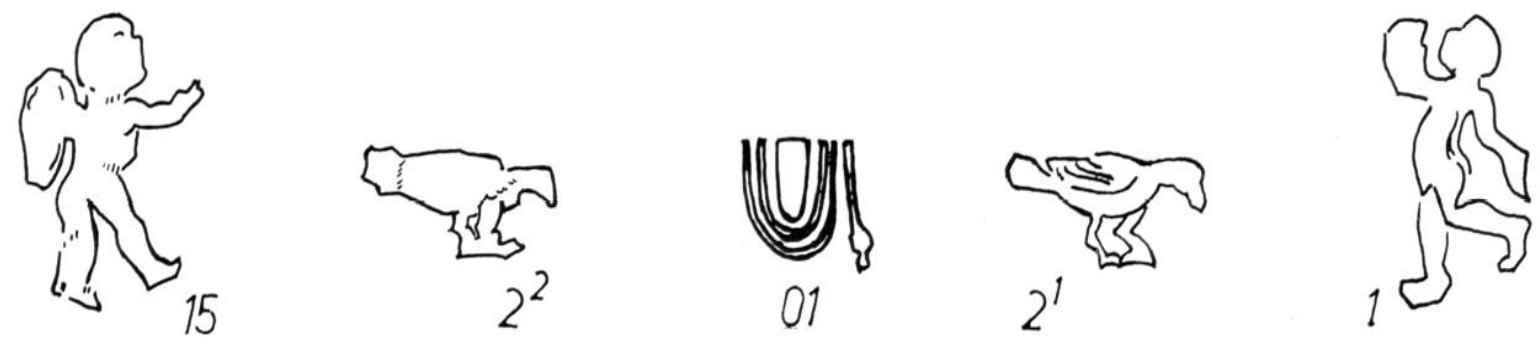

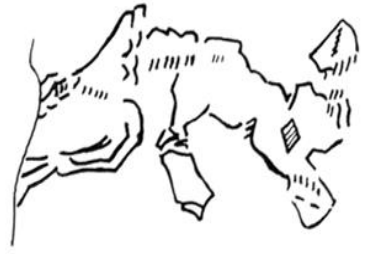

16
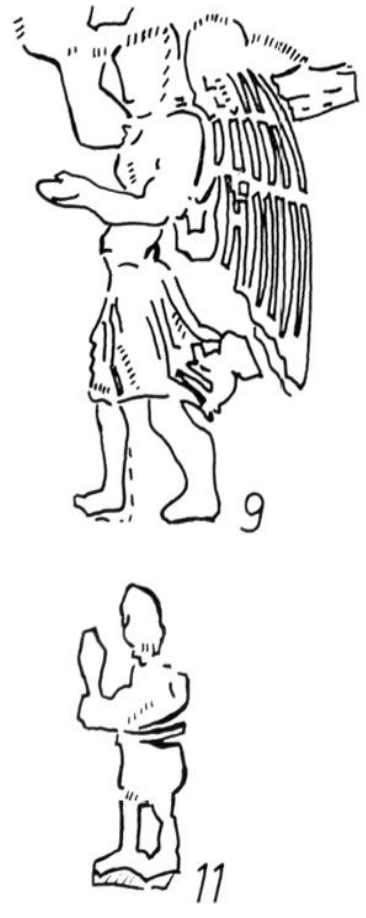

19

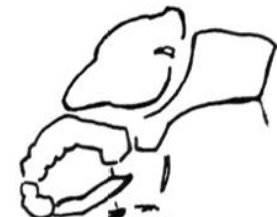

(iii)
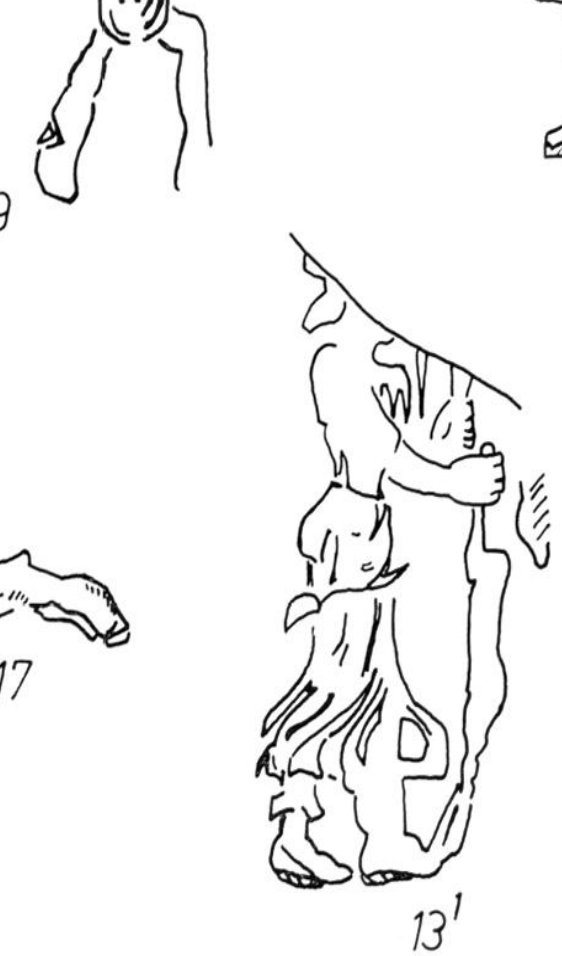

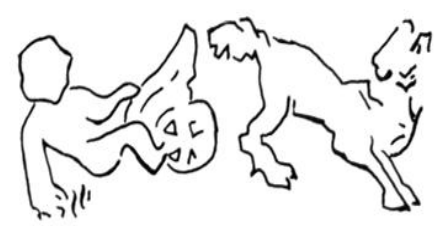

$16 b$
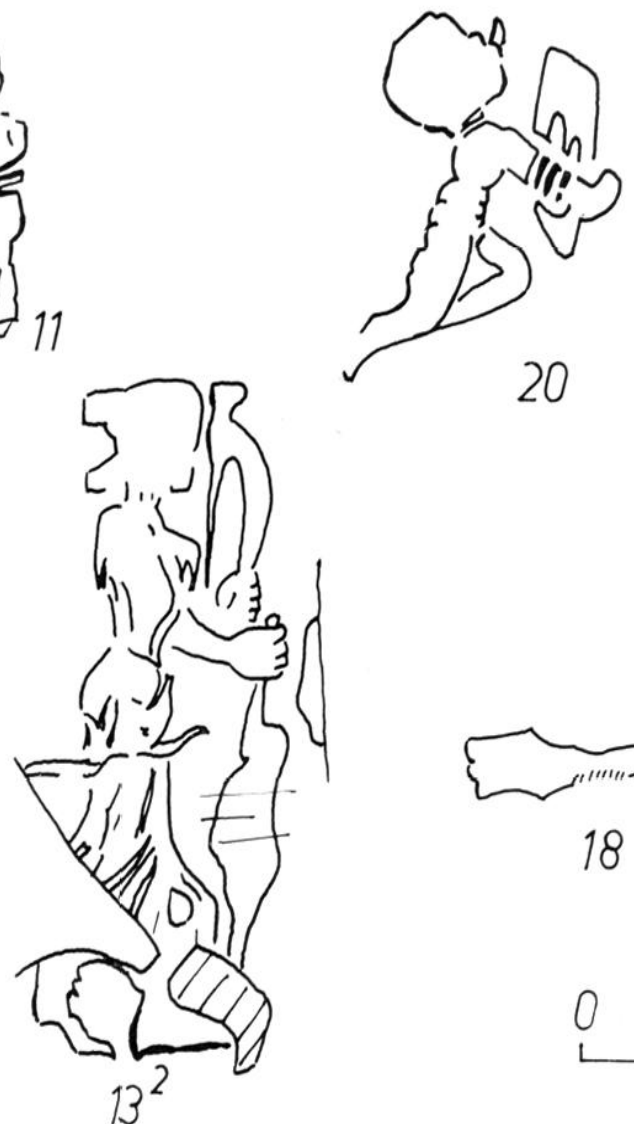
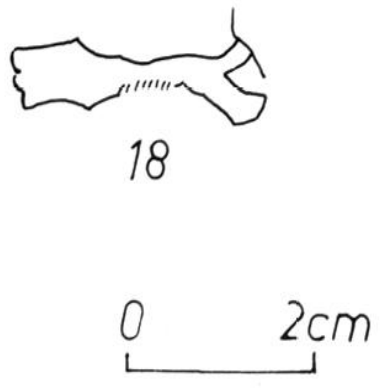

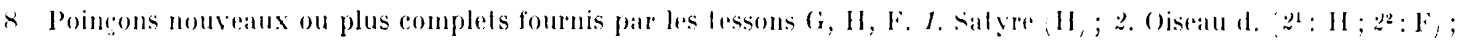

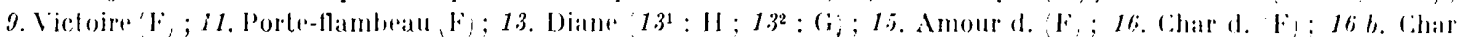

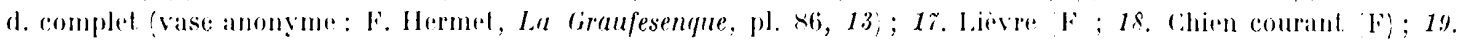

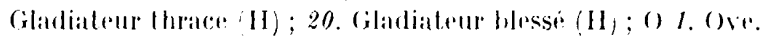




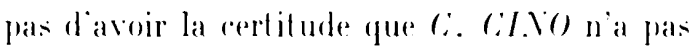
ulilise des motifs semblables el opposes l'un a l'aulre. Rien. en tout ras. dans res nouveaux échanlillons, ne nous détourne de penser que sur ces bols, commesur les premiers les mélopes semblables. ocrupant la mime place. par rapport à un axe ou a un centre de symétrie. renfermaient le mème décor.

si nous dressons le calalogue des motifs. nous voyons que rertains d'entre eux, dejoj reconnus mais incomplets. apparaissent. sur le bol Fe dans leur entier : la Victoire. le portellambeau !fig. $x$. 9 et 11: Oulre res details. le mome vase revele des lypes nouveaux : mon aurige jele a bas de son char fig. 9. 2: 8.16 el 16 b), un Amour marrhant vers la droite. un licvere ef un chien courants (tig. s. 15. 1\%, 18 . le bol tl presente. sans mulialion relle fois. la silhouette du petit salyre. une empreinte plus parfaile de l'oiseau tourné a droile et un exemplaire presque romplet de la Diane (fig. 8. 1.21 . 1.31 J. Vais, surtout. deux poingons inconmus dans le répertoire de $(: I . V)$ so répilent dans deux grandes métopes: un couple de gladiateurs (fig. $x .19$ et 20 ; employés dejoi par M. CRESTIO el BIRACILLVS. I. tableau des élements ici ulilises el de leurs analogues (p. 215) ronfirme, pour les nouveaux bols, les ronclusions qui s'étaient dégagées de lexamen des quatre premiers vases. en parliculier la resiemblance frappanle avec le reper-

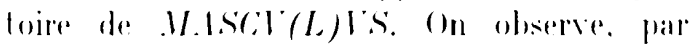
ailleurs, le caractiere see at deponille de l'ormementalion. qui peul sexpliquer par la dalalion tardice dejà proposée : extreme fin du ger. oul

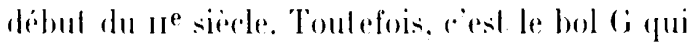
apporte la nouveaute la plus interessanle : oul re un exemplaire de la blane légerement

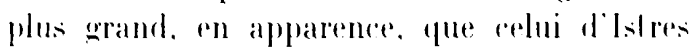

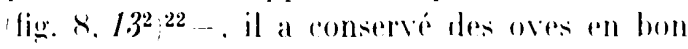

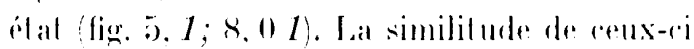
avere le tye de lowe 1 de Banassale est perd

22 Ja forme partiruliere du poincon est identigue mais la hanteur esl legrement differente. Il pourrait sacrir de deus matrices dont l'une serail reduile par surmoulage les aul res poincons sont de taille constante. llais fést sans doule simplement le resultat d'ume impression plus on moins profonde dans les momles. Carr il ne semble pas, d'apries les frottis de linlimille four toules les proportions soirnl egalement modifions. rontestable ${ }^{23}$. Celte derouverte montre que la production de $C: I$ ( ) largement inspirée en particulier. du répertoire de M.LSC:I $(L) 1 \mathrm{~S}$ el récianl. au moins une fois ${ }^{24}$. l'emploi de love a stand. a $u$ carartiore apparemment hybride puisque les produits decores de:

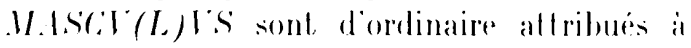
la Ciraufesenque, bien que re potier semble romnu a Banassare all moins par des vases lisses ${ }^{25}$. Il convient dies lors de définir exactement les questions que posent la silualion de CINO). On doit se demander d'abord si la présence de love "raraclerislique de Banassar $\|^{26}$ est rontraignante el si ce dernier n'a été utilise. comme le pense Cl. Morelel. que dans re centre. si ce jugement se confirme, C: Cil.Vo). inlluenere par des potiers connus dans l'atelier rabale el lablier rutione, aurait produit surement au moins une partie de ses moules decorés a Banassac. Sinon. l'hésitation resle permise entre les deux centres. pour l'ensemble de sal fabrication, lant qu'une elude approfondie n'aura pas permis soll de reconnait re ce qui, dans l'ouve de nol re potier comme dans relle de ses confreres. est imputable a l'une ou l'autre origine 28 , soit. de confondre. au moins partiellement. le travail des deux aleliers. Dans l'incertilude oil nous nous trouvons, nous pourons. en lout ass, chereleer a préciser re quont appris les comparaisons que nous avons faites. Valgre lonfluence prédominante

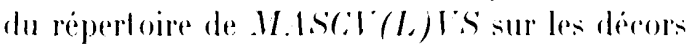
de (II.T) il apparail que l'analyse de l'ensemble des lessons revile des rapports aver un grompe de potiers pluliot quarer l'un ou

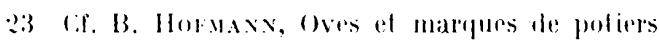
de Bamassac Foublles 1961-1961, dans Rei Crelariae

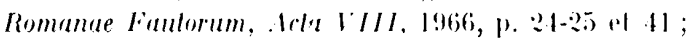

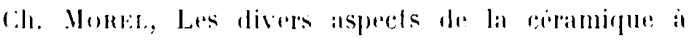

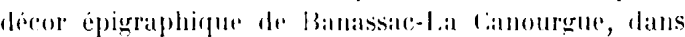
liei Cretariae liomanue liauloram, Arta $I I I, ~ I 9 t 1$, 1. 16 .

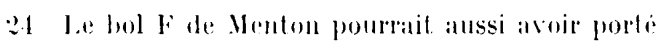
cot ove cit. infra, dig. 9,2 at 3 .

25 In potier..., 11. 73. Sur la production de.

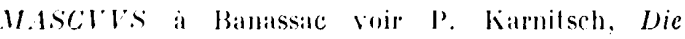
Reliefsigillata ron Ovilana, J.jn\%, 1959, 1). 27.

26 13. Howaxi, op. cil., p. $\because 1$.

27 l,or. cil.

is Voir, par remple les queslions poseres par B. Hofmann a propos des rapports do RIR.ACILILI's avec la cimafesenclue op. cil., p. 31 ! 
l'autre d'entre eux. Les plus fréquemment cilés sont MASCV (L)VS, WERCATOR, BIRACILLVS, L. COSIVS VIRILIS, mais le petit nombre des tessons ronservés et le mauvais étal des pieces identifiées ne permet pas d'établir, par exemple. un ordre de succession dans ces inspirations diverses. Certains de ces fabricants sont connus principalement à Ia Graufesenque, les autres a Banassac ; pourtant il faut noter qu'on les signale tous dans les deux centres ${ }^{29}$ et que l'on a souvent souligné des rapports entre eux. Par exemple le mème décor apparaîl sur deux vases signés différemment ${ }^{30}$, la communauté de style est telle qu'on peut prêter le même vase anépigraphe à MERCATOR ou a MASCV L L VS $S^{31}$, ou encore, on rencontre a la Graufesenque des moules anépigraphes ornés exactement d'un décor de BIRACILLVS ${ }^{32}$. Aussi certains auteurs contemporains ${ }^{33}$ parlent-ils d'un « groupe de potiers" qui, a l'époque de Domitien. rassemble. entre autres MERCATOR, BIRACILLVS. MASCV(L)VS. La question serait de définir ce qu'est ce groupe : s'agit-il simplement de l'ensemble de ceux qui. à la même époque, sous l'influence du plus inventif d'entre eux -- peut-être MASCI L L VS -employèrent les mèmes motifs décoratifs? Nous trouvons-nous la, plutiot, devant une collaboration organisee. durablement ou de façon temporaire, entre deux ou plusieurs fabricants? Il ne nous est pas possible de répondre à cette question. mais nous voyons au moins que C. CINO parait ètre une sorte de reflet ou d'émanation de ce groupe, sans que nous sachions si ses rapports avec lui sont directs ou lointains, s'il s'est contenté de suivre une mode,

29 Lin potier..., p. 200. II importerait d'ailleurs de savoir si les vestiges decouverts prourent indubitablement le travail sur place des potiers ou décorateurs, car il put y avoir des echanges de malériel ou de vases entre les deux centres.

30 In potier..., n. 57, à propos de deux bols signess l'un par BIRACILLVS l'autre par MASC'V(L.)VS et publiés par R. Knorr.

31 Ibid., p. 204.

32 Cf. B. Hormaxi, loc, cit.

33 E. Ettuingen, op. cit. p. 55 at $6 x$. ou s'il elait en relation soil aver l'un des membres du groupe soit aver l'équipe, a supposer qu'elle ail existé. Iélerminer la place exacte de notre potier parmi res quelques fabricants de la Gaule du sud sera done le bul qua longue échéance il faudra proposer à notre enquete. Hais un tel travail ne saurait se passer de préliminaires el relui-ci a toul à gagner d'une meilleure connaissance du potier lui-mème.

Les nouveaux tessons -- dont nous navons pu étudier que l'épigraphie et le décor - ont permis sur ce point d'intéressants progres : la signalure co cino s EVOVIRI, en mème lemps qu'elle résoud le probleme posé jusqu'alors par le second cognomen, révale un usage encore inconnu dans ce type d'inseription : l'examen du bol F fait apparaitre un nouveau mode de construction du décor, qui pourrail ìtre une forme simplifiée du procédé précédemment mis en évidence: enfin. le répertoire connu de CINO est maintenant plus large et la question de ses rapports avec Banassac et la Ciraufesenque en apparence plus exactement définie. Il importe desormais, afin de fixer la place de CINO parmi ses ronfreres, de poursuive l'etude stylistique : préciser la composition des décors. pour en déterminer les principes et les variations et trouver, autant que possible, la raison de celles-ci accidentelle. lechnique, rhronologique?). I.e bol F. par exemple. pourrait devoir la simplicité de son ornemenlation a une fabrication tardice ou hative. Mais la mauvaise qualité d'un unique exemplaire (fig. 9 manifeste des defauts inconteslables dans le travail du potier surimpressions (fig. 9, 1 et 3), écrasement des oves (fig. 9, 2)) sans permettre d'affirmer que l'absence de modelé des molifis est due a l'emploi abusif par l'artisan, d'un moule usé. plutot qu'à l'utilisalion. par le décorateur, de poinçons défectueux. Notre materiel. en effet, est actuellement trop pauve pour qu'on puisse tirer des conclusions solides de la comparaison de vases différemment ornés. De mème, les limites du répertoire de C. CINO sont encore inconnues et la valeur des rapprochements que nous avons déja faits a besoin d'ètre éprouvée. Il est donc souhailable de rassembler le plus grand nombre 

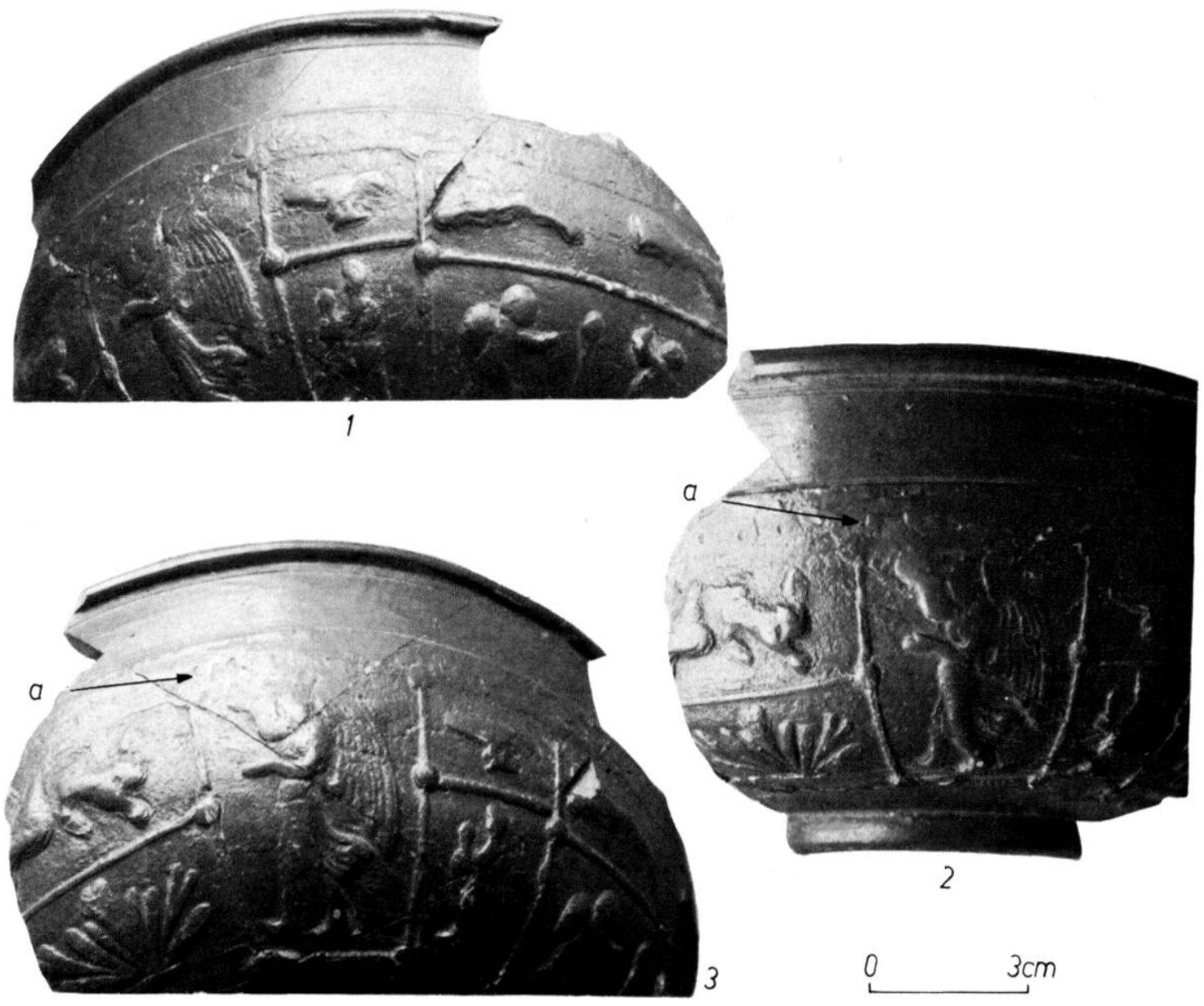

9 Le bol F: defauts lechniques. 1, noter la surimpression du lapin, du chien el lecrasement de toute la partie superieure du décor; 2 , noter le maurais etat de la représentation des cheriatux ef du char et, en a, la trace possible d'un ove a gland; 3, noter la surimpression de la Victoire al, in a, une autre vue du mème ove ecrass.

possible de témoins de la production de ret artisan. Ia plupart des pieces inventoriées se groupent actuellement a proximité de la coile méditerranéenne, mais la découverte d'un fragrment à Augrst invite à s'interroger sur lo caractiere plus ou moins fortuil de la presence d'un tesson encore isole el surtout commande d'alargir le rhamp des investigations.

Golette BínoNT. 
- Abrevialions du lableau:

$1 \quad$ : vase de (acos.

1. : : autres vases de coNo.

(;) : La Graufesenque.

1) : Banassac.

... : localisation qui niest pas explicitement, on implicitement, comprise dans da reference fournie.

Arkinsox : D. A. Atkinson, A Hoard of Samian Ware from Pompri, dans ,J.E.S., IV, 1914.

Bюxкв : D. Benker, Römische Funde in der siammlun! des hislorischen Vereins zu Giünzbur!!, Günzburğ, 1907 .

Dichemetr: : J. Dechelelle, les vases ceramiques ornes de It Gaule Romaine, Paris, 1901.

Droxis : W. beonna, Vases gallo-romains à glacure rouge et a decor moule, trouves a ciencive, dans Inz. für schureiz. Allertumskiunde, XXVIrXXIX, 1925-1928.

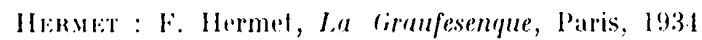

Jakobs : J. Jakohs, sigillatafunde aus einem röm. Keller zu Brege'nz, dans Jahrbuch für Allerlumsliunte, $11,1912$.
Kinvissch : P. Karnitsch, Die Reliefsigillala ron Oriland, Linz, 1959.

Кхони, Cannslall : 1. Knorr, Die derzierten Terr'l sigillata riefässe ron limnstall und limingen(irinario, stuttgarl, 1905.

Kомкв, Rollueil, 1907 : Die rerzierlen Terra sigillata lipfässe cron Rollureil, Slull gartl, 1907.

Kуокr, Rollueil, 1912 : Sü̈lyallische Terra sigillalı Gefösse von Iollureil, Stutlgart, 191:.

Kivonn, T.u.F. : Töpfer und Fabritien verzierten Terra Sigillatu des ersten .Jahrhunderts, stutlgart. 191!9.

kivonn, T.-s.-1;. : Terra-sigillalu-liefässe des erslen Jahrhunderts mil Tüpfernumen, Stull gart, 195?.

1.. .1. : London VIuserum.

(1) u.1) : F. Oswald. Inder of Fignure-lypes on Terre sigillata "Samian It are". T.iverpool, I!366-37i Jomdres, 1964.

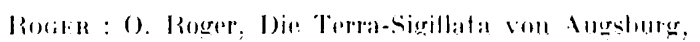
datus Zeitschr. 1. hist. Lereins für schuraben und Venburg, 33, 1907.

- Fa jalique : sujols on refierences pricédemment ciles, en partienlier dans l in polier..., p. 203-20). 


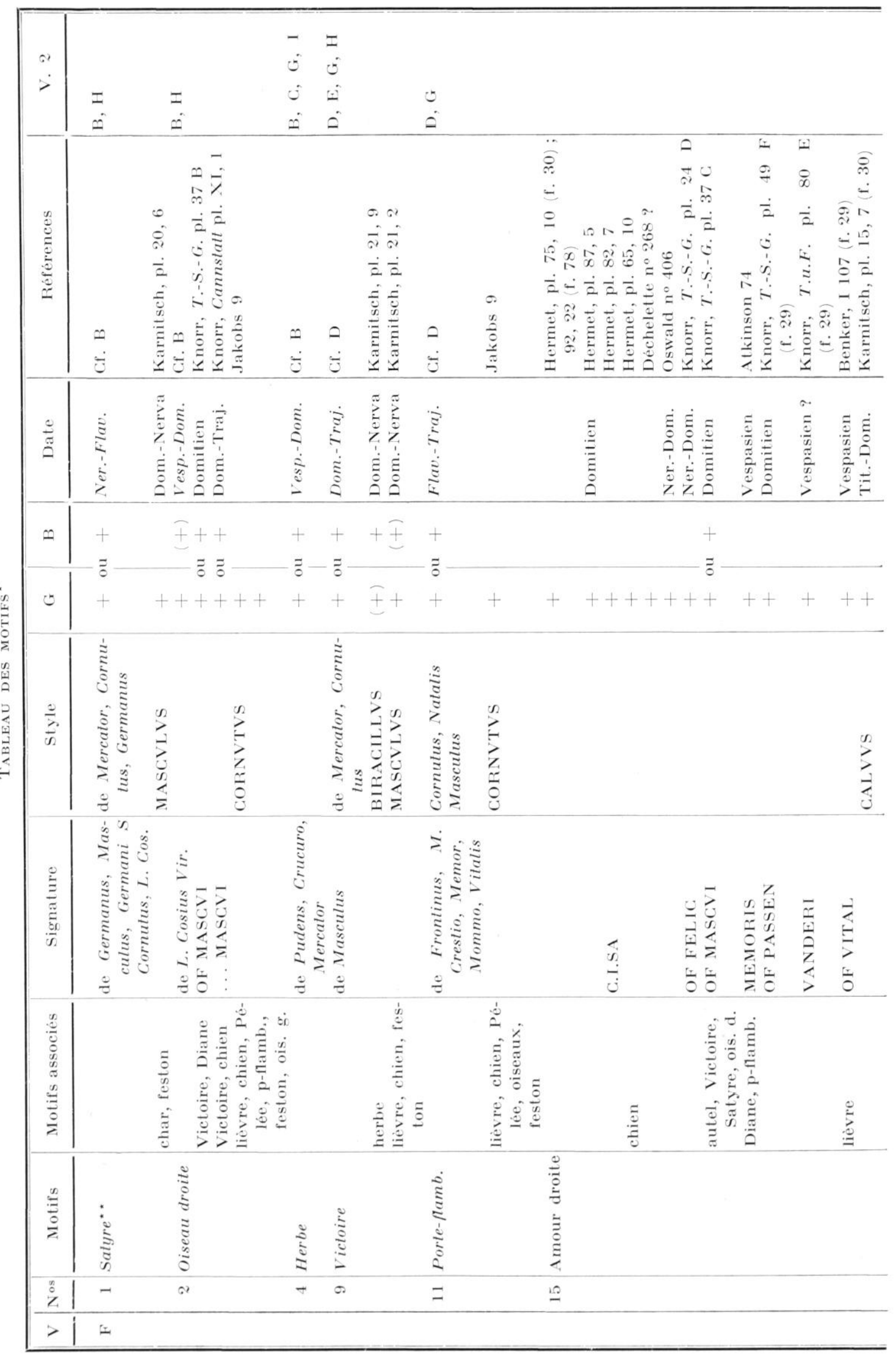




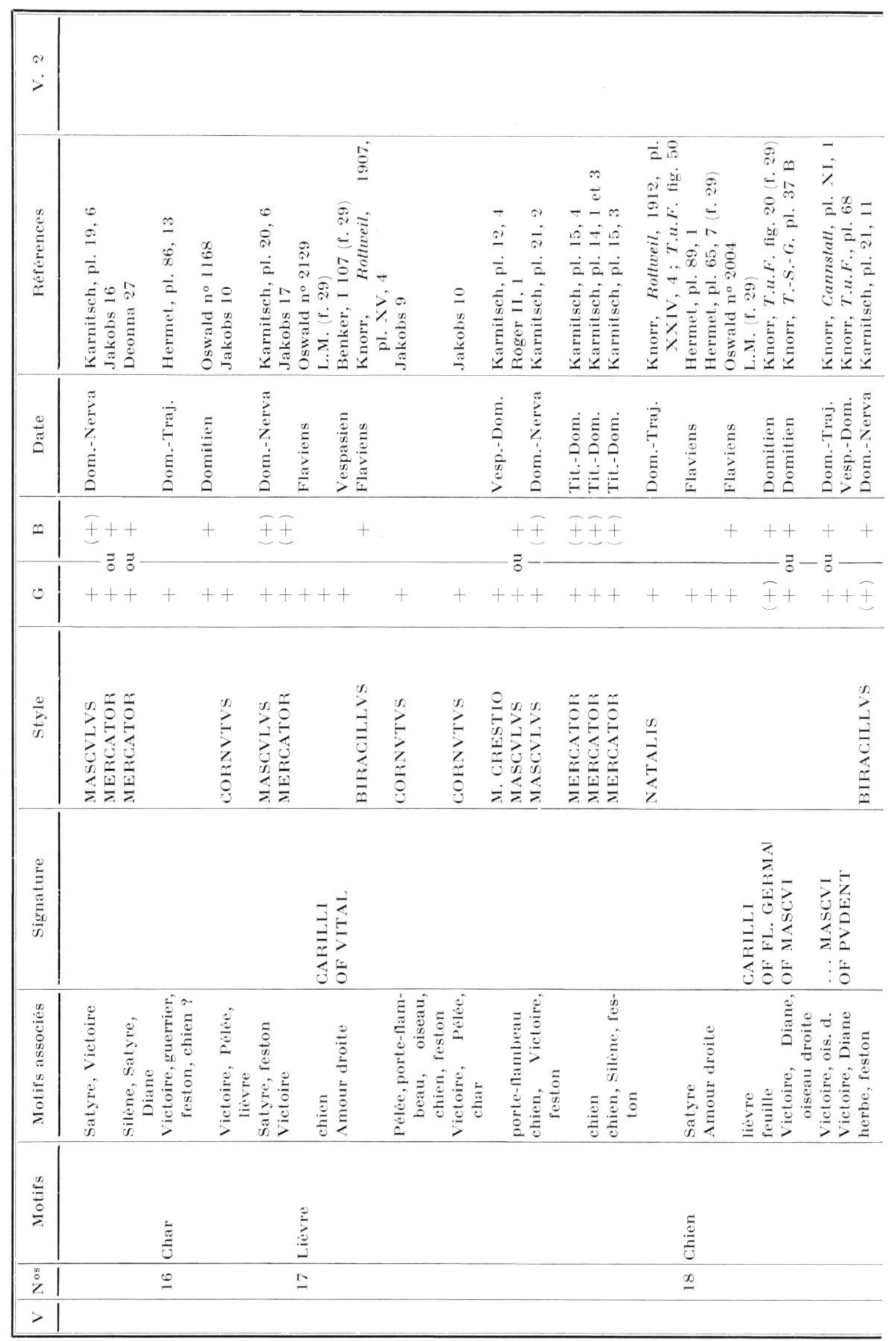




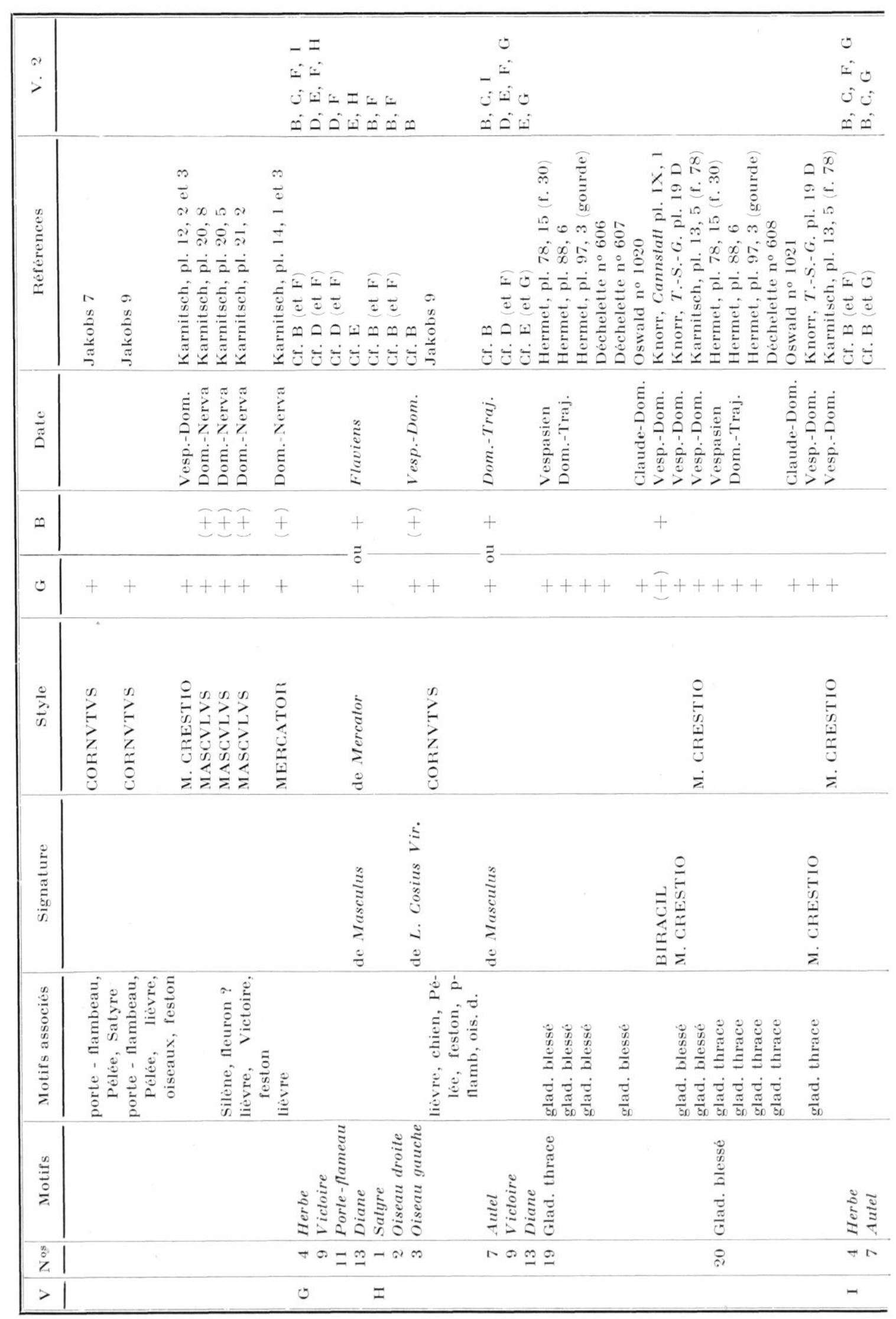

